\title{
Investigation of I-V characteristics and heat generation of multiply connected HTS conductors in parallel
}

\author{
H. C. Park ${ }^{1}$, S. Kim ${ }^{1}$, J. Cho ${ }^{2}$, M. H. Sohn ${ }^{2}$ \\ ${ }^{1}$ Department of Mechanical Engineering, Changwon National University \\ ${ }^{2}$ Korea Electrotechnology Research Institute
}

Received 27 March 2012; accepted 11 May 2012

\begin{abstract}
With continuous development of the 2nd generation HTS conductor, the critical current of the conductor is also increasing. However, many applications require more than 2 conductors in parallel to transport large current. Applications such as HTS power cables and some HTS current leads usually need much larger transport current than that provided by a single conductor and they require more than several tens of HTS conductors. In the case of parallel connection of multiple HTS conductors, the current distribution depends on the contact resistance of each conductor at the terminals for DC operation. The non-uniform distribution of the terminal resistances results in a non-uniform distribution of the current. The resultant current non-uniformity affects on the measurement of the I-V curve and the thermal performance of the multiple conductors. This paper describes the I-V curves obtained from multiply connected HTS conductors with different terminal contact resistances to investigate the relationship between the distorted I-V curve and heat generation.
\end{abstract}

\section{INTRODUCTION}

The critical current of the HTS conductor is increasing with the continuous development of the 2nd generation HTS conductor. However, it is necessary to connect several HTS conductors in parallel to increase the transport current above the critical current of a single conductor. In general, several HTS conductors are used for HTS current leads [1]-[2] and several tens of HTS conductors are used for HTS power cables [3]-[4]. Some HTS power cables and current leads require transport current of more than $10 \mathrm{kA}$.

In the case of DC operation, the current distribution is affected by the terminal contact resistances between the multiple conductors while the distribution is also affected by the self and mutual inductance in AC operation [5]-[6].

Due to the non-uniform current distribution, the measured I-V curve of multiply connected conductors can be affected by the configurations of the voltage taps unlike the measurement of a single conductor. Consequently, it is the measured $\mathrm{I}-\mathrm{V}$ curve can show additional resistive components or negative voltage and it can lead to inaccurate prediction of heat generation and thus an incorrect analysis [7]-[8].

\footnotetext{
* Corresponding author: seokho@changwon.ac.kr
}

Moreover, current redistribution also occurs as the transport current approaches the critical current due to the resistive component of the HTS conductor and it generates additional heat loss.

This paper describes the analysis results of two-strand and multi-strand HTS conductors according to various configurations of the voltage taps. The distortion of the I-V curves and the calculated heat generation are presented.

\section{ANALYSIS OF CURRENT DISTRIBUTION}

\subsection{Two strand analysis model}

For a simple analysis of the distorted I-V curve, which depends on the configuration of the voltage taps, a two strand analysis model is suggested.

Table 1 shows the specifications of the HTS conductor. Each HTS conductor has the same critical current and index value. Fig. 1 shows the circuit diagram of the two strands model with different contact resistances. Four kinds of voltage taps of $\mathrm{V}_{1}$ to $\mathrm{V}_{4}$ were considered.

The current distribution and the voltage drops of the circuit diagram can be calculated by considering the variable resistance of the HTS conductor, which is determined by the transport current, as in (1) to (3).

TABLE I

SPECIFICATIONS OF HTS CONDUCTOR USED IN TWO STRAND ANALYSIS.

Length $(\mathrm{cm})$

Critical current (A)@77 K, self field

Index value

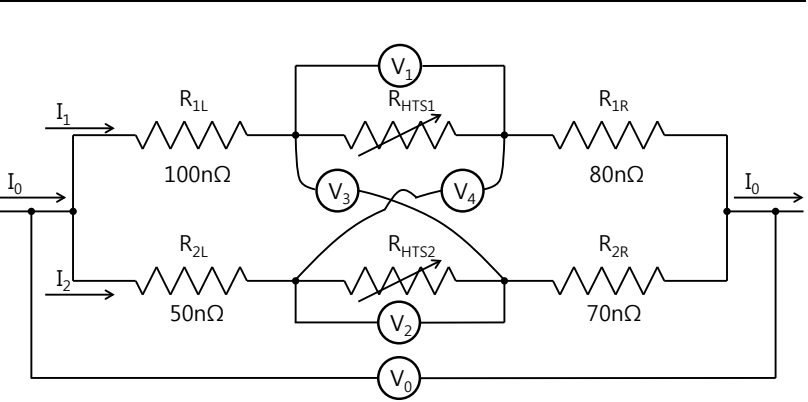

Fig. 1. Circuit diagram of two strands model. 


$$
\begin{aligned}
& I_{0}=I_{1}+I_{2} \\
& V_{0}=\left(R_{1 L}+R_{1 R}+R_{H T S 1}\right) I_{1}=\left(R_{1 L}+R_{1 R}\right) I_{1}+l E_{0}\left(\frac{I_{1}}{I_{1, c}}\right)^{n} \\
& V_{0}=\left(R_{2 L}+R_{2 R}+R_{H T S 2}\right) I_{2}=\left(R_{2 L}+R_{2 R}\right) I_{2}+l E_{0}\left(\frac{I_{2}}{I_{2, c}}\right)^{n}
\end{aligned}
$$

Where $\mathrm{I}_{0}, \mathrm{I}_{1}$, and $\mathrm{I}_{2}$ are the total transport current and the current at conductor 1 (HTS1) and conductor 2 (HTS2). $\mathrm{V}_{0}$ is the total voltage drop through the terminal resistances and HTS conductors. $\mathrm{R}_{1 \mathrm{~L}}, \mathrm{R}_{1 \mathrm{R}}, \mathrm{R}_{2 \mathrm{~L}}$, and $\mathrm{R}_{2 \mathrm{R}}$ are the terminal resistances shown in Fig. $1 . \mathrm{E}_{0}$ is the critical voltage of $1 \mu \mathrm{V} / \mathrm{cm}$ and $l$ is the length of the conductor in centimeters. $\mathrm{I}_{1, \mathrm{c}}$ and $\mathrm{I}_{2, \mathrm{c}}$ are the critical current of HTS 1 and HTS2, respectively.

\subsection{Two strand analysis results}

By increasing the transport current $\left(\mathrm{I}_{0}\right)$, four kinds of voltage signals can be calculated, as shown in Fig. 2 using (4), (5). $\mathrm{V}_{1}, \mathrm{~V}_{2}, \mathrm{~V}_{3}$, and $\mathrm{V}_{4}$ correspond to the voltage taps in Fig. 1.

$$
\begin{aligned}
& V_{3}=\left(R_{1 R}+R_{H T S 1}\right) I_{1}-R_{2 R} I_{2}=R_{1 R} I_{1}+l E_{0}\left(\frac{I_{1}}{I_{1, c}}\right)^{n}-R_{2 R} I_{2} \\
& V_{4}=\left(R_{2 R}+R_{H T S 2}\right) I_{2}-R_{1 R} I_{1}=R_{2 R} I_{2}+l E_{0}\left(\frac{I_{2}}{I_{2, c}}\right)^{n}-R_{1 R} I_{1}
\end{aligned}
$$

As shown in Fig. 2, $\mathrm{V}_{1}$ shows a large index value curve and $\mathrm{V}_{2}$ shows a small index value curve. In the case of $\mathrm{V}_{3}$, it shows a negative voltage drop and $V_{4}$ shows initially resistive voltage.

These results are caused by the non-uniform current distribution. Since the terminal resistance of HTS2 is smaller than that of HTS1, more current flows through HTS2 than HTS1. However, as the current at HTS2 increases and approaches the critical current, it becomes resistive and the current starts to bypass to HTS1. This results in small and large index value curves. Therefore, it is thought that extraordinary I-V curves can be obtained because the measured I-V curves of the two strand

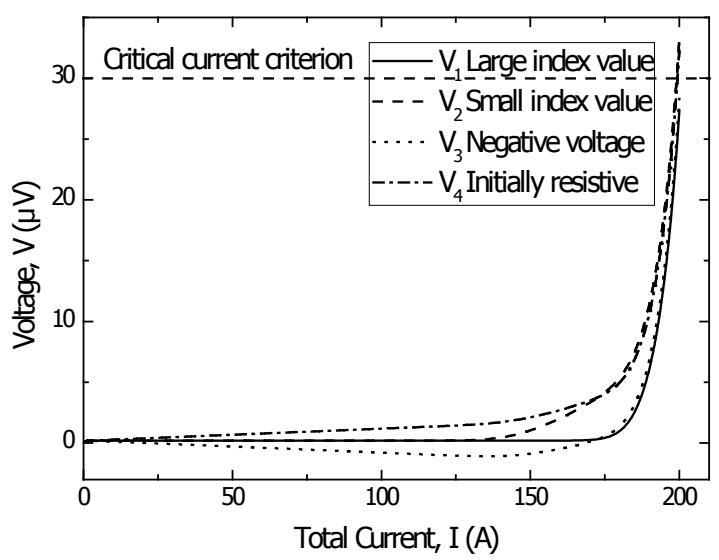

Fig. 2. I-V curves for each configuration of the voltage taps.

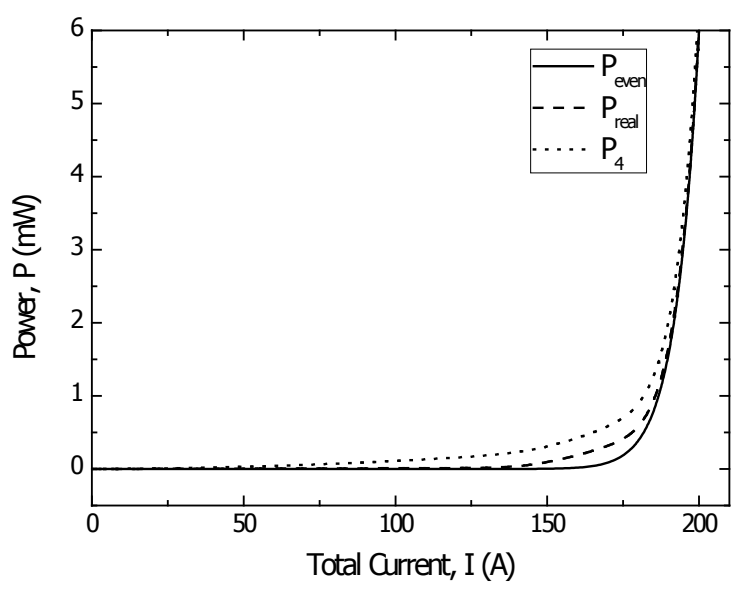

Fig. 3. Heat generations based on each calculated voltage drop.

conductor depend on the configuration of the voltage taps, as shown in Fig. 2.

With each calculated voltage signal, the heat generation can be calculated. If we select $\mathrm{V}_{4}$ as the representative voltage signal, the heat generation at the two strand HTS conductor can be calculated by simply multiplying the total transport current with the voltage, as in (6). However, the true heat generation can be calculated by considering each voltage drop and individual current, as in (7). Moreover, heat generation of an even current distribution can be calculated by assuming equal terminal resistances, as in (8).

$$
\begin{gathered}
P_{4}=V_{4} \times\left(I_{1}+I_{2}\right) \\
P_{\text {real }}=P_{1}+P_{2}=V_{1} \times I_{1}+V_{2} \times I_{2} \\
P_{\text {even }}=l \times E_{0}\left(\frac{I_{0}}{2 \times I_{c}}\right)^{n} \times I_{0}
\end{gathered}
$$

Fig. 3 shows the calculated heat generation based on each calculation method. As shown in Fig 3, $\mathrm{P}_{\text {even }}$ is the smallest and $\mathrm{P}_{4}$ is the largest.

Among the calculated heat generation variables, $\mathrm{P}_{\text {real }}$ is the actual heat generation of the two strand conductor model. However, the results show that the calculation results in an incorrect prediction according to the configuration of the voltage taps. Moreover, it is seen that additional heat generation occurs through current redistribution compared with the case of even distribution with equal terminal resistances.

\subsection{Multi-strands analysis model}

In the case of HTS power cables and some HTS current leads, a number of HTS conductors are connected in parallel to transport large current. Fig. 4 shows the circuit diagram of a multi-strand conductor and Table 2 shows the specifications of the HTS conductor used in the analysis.

For the distribution of the terminal resistances, a Gaussian distribution of the resistance was used with an average value of $0.55 \mu \Omega$ and a standard deviation of 275 $\mathrm{n} \Omega(4 \sigma)$. In the analysis, the range of the resistance was limited within 0.1 to $1 \mu \Omega$. Fig. 5 shows the terminal resistances generated by the Gaussian distribution. 


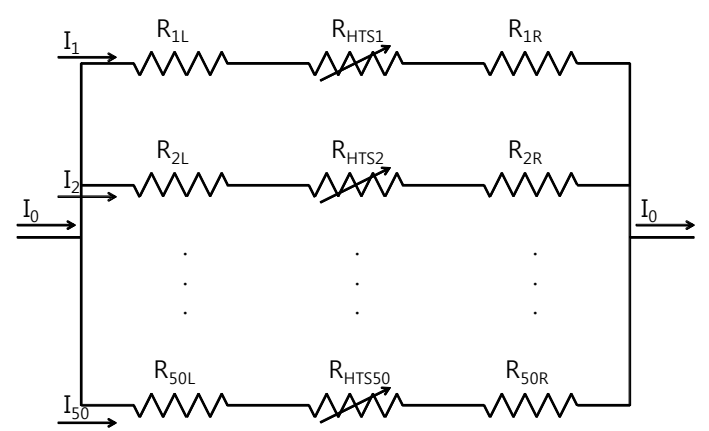

Fig. 4. Electric circuit for multi-strand HTS conductors in parallel.

TABLE II

SPECIFICATIONS OF HTS CONDUCTOR USED IN MULTI STRAND ANALYSIS.

\begin{tabular}{ll}
\hline Critical current $(\mathrm{A})$ & 100 \\
No. of conductor (EA) & 50 \\
Length $(\mathrm{m})$ & 2 \\
Contact resistance $(\mu \Omega)$ & $0.1 \sim 1$ \\
\hline
\end{tabular}

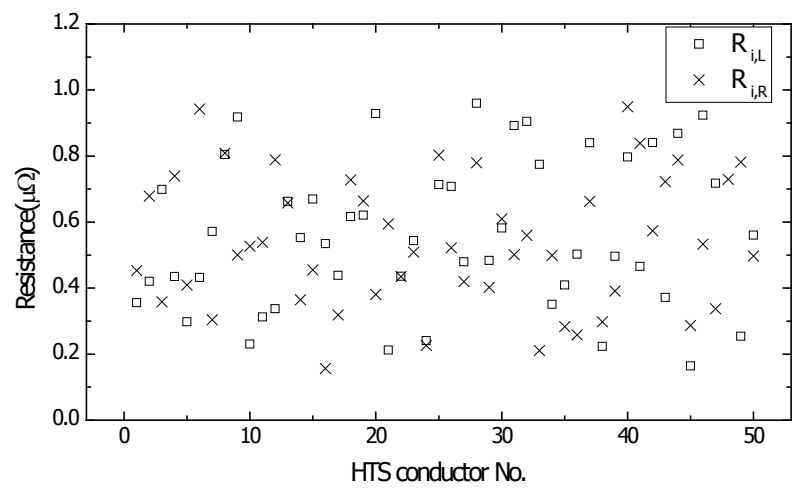

Fig. 5. Distribution of terminal resistances for multi-strands HTS cable in analysis.

To analyze the current distribution, voltage drops and heat generation, (1), (2), and (3) were expanded for 50 HTS conductors.

\subsection{Analysis results of multi-strand model}

The analysis is performed by calculating the current distribution at each step of the total transport current. Similar to the results of Fig. 2, various voltage curves can be obtained according to the configurations of the voltage taps.

The obtained voltage curves can be divided into the following groups.

\section{Negative voltage graph}

2. Initially resistive graph

3. Large index value graph

4. Small index value graph

Fig. 6 shows a negative voltage curve and an initially resistive voltage curve with the standard curve of an even current distribution for comparison.

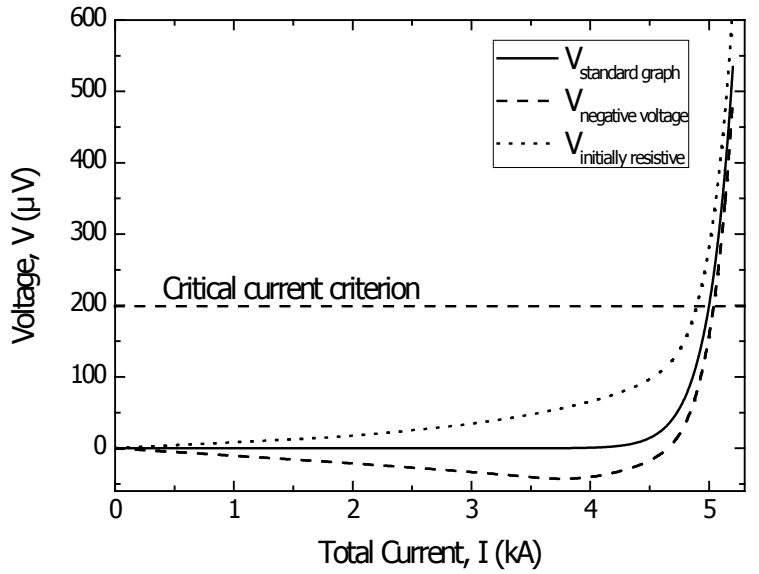

Fig. 6. Calculation results of initially resistive and negative voltage curve.

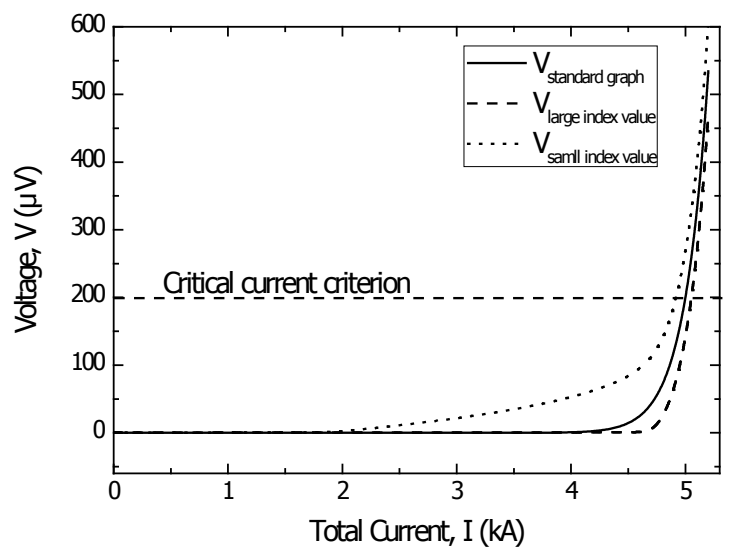

Fig. 7. Calculation results of large index value and small index value curve.

Fig. 7 shows the large index value curve and a small index value curve with the standard curve. $\mathrm{V}_{\text {large index value }}$ and $\mathrm{V}_{\text {small index value }}$ correspond to the voltage drop through the HTS conductors of the largest and the smallest terminal resistance, respectively. These configurations are similar to $\mathrm{V}_{1}$ and $\mathrm{V}_{2}$ in Fig. 1.

As shown in Figs. 6 and 7, various I-V curves can be obtained according to the various configurations of the voltage taps. The results can be explained in a similar manner as presented for the two strand model.

Using the obtained voltage curves, the heat generation can be calculated by the following equations. First, the heat generation can be calculated by assuming an even distribution, as in (9). Based on the initially resistive voltage curve, the heat generation is calculated as delineated in (10). However, the real heat generation should be calculated by considering each distribution of the current and voltage drops as in (11).

$$
\begin{aligned}
& P_{\text {even }}=l \times E_{0}\left(\frac{I_{0}}{50 \times I_{c}}\right) \times I_{0} \\
& P_{\text {initially resistive }}=V_{\text {intitally }} \text { resistive } \\
& P_{\text {real }}=\sum_{\text {tatal }}^{50} V_{n} \cdot I_{n}
\end{aligned}
$$




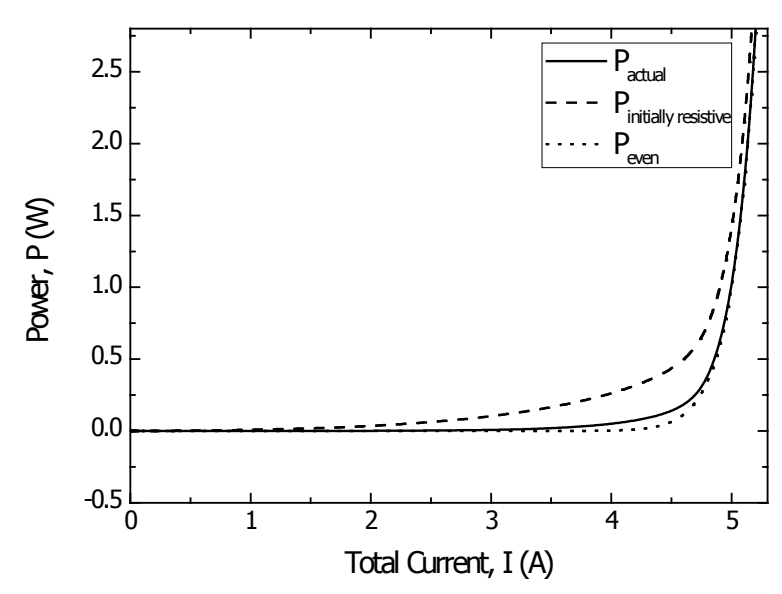

Fig. 8. Calculation heat generation based on different calculation methods.

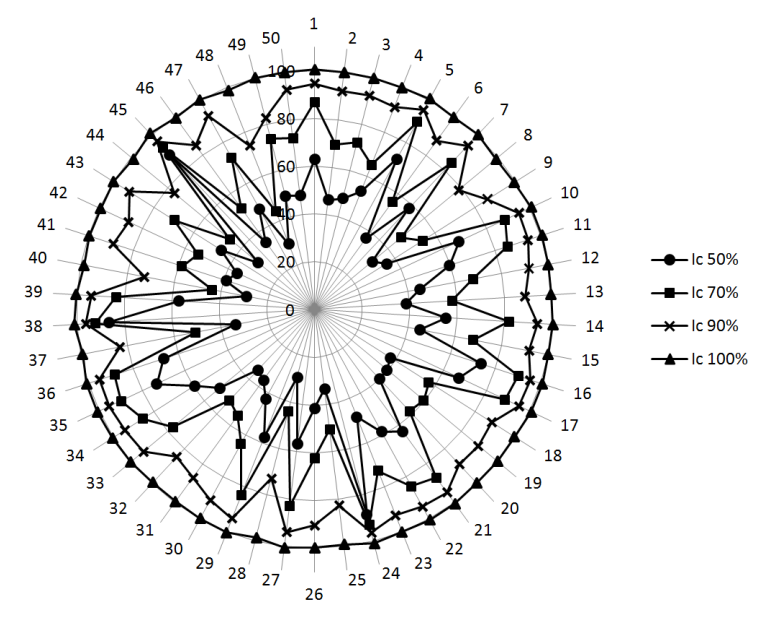

Fig. 9. Variation of the current distribution for different total transport current.

Fig. 8 shows the calculated heat generations by each calculation method. As shown in Fig. 8, the heat generation is minimized for the even distribution, while larger heat generation is obtained with the initially resistive voltage curve. Moreover, $\mathrm{P}_{\text {real }}$ also shows slightly larger heat generation.

These calculation results indicate that the experimental results from a short sample HTS power cable depend on the configurations of the voltage taps, and the results cannot be directly used to calculate the expected heat loss below the critical current.

Fig. 9 shows the variation of the current distribution for different total transport currents. Initially, the current distribution depends only on the distribution of the terminal resistances; however, the current distribution becomes uniform as the transport current increases.

During this process, additional heat loss is generated through current redistribution when the transport current approaches the critical current. The amount of heat generation depends on the non-uniformity of the terminal resistance and the length of the HTS conductors, because over current is limited by the increased resistance at the HTS conductors and it is redistributed through the terminal resistances. Therefore, the over current can be easily bypassed to other HTS conductors if the resistances among the conductors are small.

\section{CONCLUSION}

In this paper, some problems related to distorted voltage signals were investigated with a simple analysis of the electric circuits of two strand and multi-strand HTS conductors. It was verified that the distorted voltage signal can result in incorrect prediction of the heat generation. Therefore, the I-V curve for multiply connected HTS conductors should be carefully interpreted.

To suppress the distorted voltage signal, an even distribution of the terminal resistance is necessary. However, some dispersion of the resistance is inevitable, since the jointing process is carried out manually and it involves various uncontrolled effects such as soldering surface, solder thickness, and so on. Investigation focused on precise measurement of the current distribution is required to better predict the actual heat generation of a multi-strands conductor.

In addition, the use of several additional HTS conductors that connect multiple conductors can be considered to minimize the heat generation from the redistribution process by reducing the resistances among the conductors.

\section{ACKNOWLEDGMENT}

This paper was supported by research funds of Changwon National University in 2010.

\section{REFERENCES}

[1] M. Sohn, S. Kim, K. Sim, J. H. Bae, S. Lee, B. Eom, H. Park, "Fabrication and characteristics of current lead with 2G HTS tapes," KIEE, Summer Annual Conference 2009, July 2009.

[2] H. Park, K. Kim, D. Kim, A. Kim, M. Park, I. Yu, S. Kim, K. Sim, M Sonh, "Design and manufacture of HTS current lead for 10kJ SMES," KIEE, Summer Annual Conference 2009, July 2009.

[3] S. Choi, S. lee, K. Sim. J. Cho and et al, "DC critical current test method for $22.9 \mathrm{kV} / 50$ MVA Superconducting Power Cable Considering the Uncertainty," IEEE Trans. Appl. Supercon, vol. 20, no. 3, pp. 1272-1275, June 2010.

[4] J. Cho, K. Sim, J. H. Bae , H. J. Kim and et al., "Design and Experimental Results of a 3 phase $30 \mathrm{~m}$ HTS Power Cable", IEEE Trans. Appl. Supercon, vol. 16, no. 2, pp. 1602-2605, June 2006.

[5] J. H. Kim, M. Park, J. Cho, K. Sim, S. Kim and I. K. Yu, "Current distribution analysis of conducting and shield layers of HTS power cable under utility fault condition", IEEE Trans. Appl. Supercon, vol. 19, no. 3, pp. 1781-1784, June 2009.

[6] J. Bae, D. K Bae, J. W. Cho, K. Sim and T. K. Ko, "Non-uniform current distribution of multi-strand HTS cable", KIEE Trans. Energy conversion system, vol. 53, no. 7, pp. 424-429, 2004.

[7] K. Sim, S. Kim, S. Lee, J. Cho and T. K. Ko, "The estimation of the current distribution on the HTS cable by measuring the circumferential magnetic field," IEEE Trans. Appl. Supercon, vol. 20, no. 3, pp. 1981-1984, June 2010.

[8] K. Sim, S. Kim, J. Cho, D. Kim, C. Kim, H. Jang, S. Sohn, S. Hwang, "DC critical curent and AC loss measurement of the $100 \mathrm{~m}$ 22.9 kV/50 MVA HTS cable," Physica C, vol. 468, no. 15-20, pp. 2018-2022, September 2008 